\title{
Flexible Nonlinear Control Technique with Applications to Power Systems
}

\author{
Mark Gordon* David J. Hill** \\ * Research Center for Electric Technology, \\ Technical University of Denmark, 2800 Kgs. Lyngby, Denmark \\ (e-mail: mag@elektro.dtu.dk). \\ ** Research School of Information Sciences and Engineering, \\ Australian National University, Canberra ACT 0200, Australia \\ (e-mail: david.hill@anu.edu.au)
}

\begin{abstract}
This paper presents the so called Direct Feedback Linearization (DFL) technique as a simple and flexible method for nonlinear control design. The DFL avoids the complexity of the well known differential geometric method, instead it uses Implicit Function Theorem (IFT) to eliminate system nonlinearities. This allows more flexibility in the exact linearization steps. To consider the effect of plant parametric uncertainties, robust control theory is used to ensure the stability of the DFL compensated system. As an example application, four different robust nonlinear excitation controllers are designed and compared to enhance transient stability of power systems. The main advantage of the proposed technique is the possibility for a control engineer to choose the most appropriate performance enhancing nonlinear compensating controller based on availability of measurements or required simplicity in the feedback loop design.
\end{abstract}

Keywords: Feedback control methods, Feedback Linearization, Power system control, Excitation control

\section{INTRODUCTION}

Power systems are large scale complex nonlinear systems. It is well known that control through the excitation loop of synchronous generator is the most effective method for improving dynamic performance and transient (angle) stability of power systems. Various types of control strategies are available based on the linearization technique employed. The conventional excitation control system design is based on approximately linearized power plant model, i.e. the single machine infinite bus (SMIB) model is linearized around an operating point and linear control theory is applied to design linear excitation controller. This type of control suffers performance degeneracy when operating conditions change. In fact linear controllers may even destabilize the system, while the operating point of the power system is changed away from the equilibrium point at which the approximate linearization is realized, see Lu and Sun (1989). Because of this, the development of stabilizing excitation systems both theoretically and practically has received considerable attention.

In recent decades, nonlinear control theory with special emphasis on the geometric feedback linearization approach has been developed and proved to be an effective design technique for control of systems described by affine nonlinear mathematical models. Isidori (1995) summarized a large literature on nonlinear control system theory. An example of early works considering the application of geometric nonlinear control to power systems can be found in Marino (1984); Lu and Sun (1989); Chapman et al. (1993). It is important to stress that geometric feedback linearization technique involves a static state feedback and special nonlinear coordinate change. As a result of diffeomorphism mapping (coordinate transformation), the physical variables can be changed to nonphysical ones, and this may result in control of variables other than what is practically required. Further more, the succeeding closed loop design can be too complex to be accepted by engineers Gao et al. (1992).

In contrast to differential geometric technique, the DFL method avoids the geometric coordinate change and control of all physical state variables can be preserved, see Gordon and Hill (2006). The DFL method can linearize the system over a very wide range, independently of the operating point of the system. Considering the effects of plant parametric uncertainties or remaining nonlinear interconnections, robust control theory is used in this paper to ensure the stability of the overall DFL compensated system. While the application of DFL has received considerable attention, there has been no emphasis on flexibility characteristics of this technique. For example, it is quite common to refer to the same control model, see controllers in Guo et al. (2001); Zhu et al. (1997); Wang et al. (1993) and the issue of practicality of this technique has been neglected.

In this paper we propose and present DFL technique as a flexible control method for nonlinear systems. As an application system, we concentrate on transient stability enhancement of power systems by means of nonlinear excitation control. One of the main problems for control/power system engineers is to obtain the most suitable expression 
for excitation control strategy that is optimal for the original nonlinear model, and secondly, to practically realize and implement control strategy based on local or available measurements. Therefore, we present DFL technique as a useful method that can offer a variety of solutions for the above mentioned problems.

\section{POWER SYSTEM MODEL}

We consider a SMIB power system model which consists of a single generator connected through two parallel transmission lines to a very large network represented by an infinite bus. A third order single axis model has been found sufficient in designing excitation system controllers, see Wang et al. (1993) A simple model can be written as follows:

$$
\begin{aligned}
\dot{\delta} & =\omega(t)-\omega_{0} \\
\dot{\omega} & =\frac{D}{2 H}\left(\omega(t)-\omega_{0}\right)-\frac{\omega_{0}}{2 H}\left(P_{e}-P_{m}\right) \\
\dot{e}_{q}^{\prime} & =\frac{1}{T_{d o}^{\prime}}\left(E_{f}-\Delta x_{d} i_{d}-e_{q}^{\prime}\right)
\end{aligned}
$$

with the following electrical equations:

$$
\begin{aligned}
P_{e}(t) & =e_{q}^{\prime} i_{q}=\frac{e_{q}^{\prime} V_{\infty} \sin \delta(t)}{x_{d \Sigma}^{\prime}} \\
i_{d}(t) & =\frac{e_{q}^{\prime}-V_{\infty} \cos \delta(t)}{x_{d \Sigma}^{\prime}} \\
i_{q}(t) & =\frac{V_{\infty} \sin \delta(t)}{x_{d \Sigma}^{\prime}} \\
Q_{e}(t) & =\frac{e_{q}^{\prime} V_{\infty} \cos \delta(t)-V_{\infty}^{2}}{x_{d \Sigma}^{\prime}} \\
V_{t}(t) & =\sqrt{e_{q}^{\prime 2}-2 e_{q}^{\prime} x_{d}^{\prime} i_{d}+x_{d}^{\prime 2}\left(i_{d}^{2}+i_{q}^{2}\right)}
\end{aligned}
$$

The parameters of the system under consideration are: $\omega_{0}=100 \pi ; D=5 ; H=4 ; T_{d o}^{\prime}=6.9 ; x_{t}=0.127 ; x_{d}=$ $1.863 ; x_{d}^{\prime}=0.657 ; x_{L}=0.4853$; and the governor is assumed to be slow acting. The physical limit of the plant is taken as $\max \left|E_{f}\right| \leq 6$. The equivalent system reactance of the whole transmission link is $x_{d \Sigma}^{\prime}=x_{d}^{\prime}+x_{t}+x_{l}$ and $\Delta x_{d}=x_{d}-x_{d}^{\prime}$. For notation description, readers are referred to Zhu et al. (1997); Guo et al. (2001). The following changes in network topology are considered:

Disturbance 1: The fault considered is a symmetrical threephase short circuit fault which occurs on one of the transmission lines at the generator $\mathrm{HV}$ transformer bus. The fault is removed by the action of a circuit breaker.

Disturbance 2: The mechanical input power of the generator has a sudden step increase of $25 \%$ at $t=t_{s}$.

\section{NONLINEAR COMPENSATOR DESIGN}

The direct feedback linearization technique offers considerable flexibility in designing nonlinear controllers. It uses the IFT to selectively eliminate specific nonlinearities. It has a simple theoretical basis but allows more flexibility than the differential geometric approach in the exact linearization steps. We consider the following 3rd order system

$$
\begin{aligned}
& \dot{x_{1}}=f_{1}(x)=a_{11} x_{1}+a_{12} x_{2}+a_{13} x_{3} \\
& \dot{x_{2}}=f_{2}(x)=a_{21} x_{1}+a_{22} x_{2}+a_{23} x_{3} \\
& \dot{x_{3}}=f_{3}\left(x_{1}, x_{2}, x_{3}, u\right)
\end{aligned}
$$

where $u$ is the input and $f_{3}$ contains the only nonlinear terms. Let the right hand side of nonlinear equation (11) be replaced by a new input $b v(t)$ as follows

$$
f_{3}\left(x_{1}, x_{2}, x_{3}, u\right)-a_{31} x_{1}-a_{32} x_{2}-a_{33} x_{3}=b v(t)
$$

where linear terms have been introduced. These can be used to preserve certain physical features. We require (12) to have a well defined solution of the form

$$
u=g_{3}\left(x_{1}, x_{2}, x_{3}, b v\right)
$$

A sufficient condition is given by the IFT, such that the solution exists if

$$
\frac{\partial f_{3}}{\partial u} \neq 0
$$

Hence (13) defines a nonlinear feedback control via which the nonlinear plant model becomes linear with respect to an arbitrary new input $v \in \Re$ in the form $\dot{x}=$ $A x+b v$ where $\mathrm{A}$ is $3 \times 3$ with elements $a_{i, j} ; i, j=$ $1,2,3$ and $b=[0,0,1]^{T}$. This idea has been applied in various ways Gao et al. (1992); Zhu et al. (1997); Guo et al. (2001); Gordon and Hill (2006) using different variables. To demonstrate flexibility here we use the model based on variables $\left(\delta, \omega, P_{e}\right)$ and allow for physical model uncertainty.

\subsection{Nonlinear Control Design 1}

From the power system model (1)-(8), we know that the generator is nonlinear through the excitation loop. To eliminate nonlinearities we differentiate (4) and combining with other equations obtain

$$
\begin{aligned}
\dot{P}_{e}= & i_{q} \frac{1}{T_{d o}^{\prime}}\left(E_{f}-\Delta x_{d} i_{d}-e_{q}^{\prime}\right) \\
& +P_{e} \cot \delta\left(\omega-\omega_{0}\right)
\end{aligned}
$$

Elimination of nonlinearities via differentiation of electrical power equation has also been applied in the previous work, for example see Guo et al. (2001); Wang et al. (1993). In this paper we propose a novel nonlinear control loop for the excitation system. By letting

$$
v_{1}=\dot{P}_{e}
$$

and selecting $\left[\begin{array}{lll}\Delta \delta & \Delta \omega & \Delta P_{e}\end{array}\right]^{T}$ as the new vector, the DFL compensated power system model can be written as

$$
\dot{x}(t)=A x+B v_{1}
$$

where

$$
A=\left[\begin{array}{ccc}
0 & 1 & 0 \\
0 & -\frac{D}{2 H} & -\frac{\omega_{0}}{2 H} \\
0 & 0 & 0
\end{array}\right] \quad B=\left[\begin{array}{l}
0 \\
0 \\
1
\end{array}\right]
$$

and $v_{1}$ is the new input. To design a controller for $E_{f}$ to stabilize the original model is equivalent to design a 
controller for $v_{1}$ to stabilize the compensated plant model (18). From (15) and (16) the nonlinear compensated law for the excitation control system can be obtained as

$$
E_{f}=\frac{T_{d o}^{\prime}}{i_{q}}\left(v_{1}-P_{e} \cot \delta \Delta \omega\right)+\Delta x_{d} i_{d}+\frac{P_{e}}{i_{q}}
$$

where $E_{f}$ is the control input for the excitation system. It can be seen from (18) that the DFL compensated system is independent of the operating points and linear over very wide range. After linearization, the linear LQR optimal control design can be applied to design optimal feedback control law (16). An effective feedback control law of the form

$$
v_{i}=-k_{\delta} \Delta \delta-k_{\omega} \Delta \omega-k_{P e} \Delta P_{e}
$$

can be developed to achieve effective transient stability enhancement and whose performance is independent of the operating conditions.

\subsection{Nonlinear Control Design 2}

The excitation field voltage term occurs naturally in (3) and we eliminate the generator electrical dynamics by choosing the new input $v_{2}$ as

$$
v_{2}=\left(E_{f}-\Delta x_{d} i_{d}-\frac{P_{e}}{i_{q}}\right)
$$

and differentiating (4) we obtain

$$
\dot{P}_{e}=\frac{e_{q}^{,} V_{\infty} \cos \delta}{x_{d \Sigma}^{\prime}} \Delta \omega+\frac{1}{T_{d o}^{\prime}} i_{q} v_{2}
$$

The compensating law can be written as

$$
E_{f}=v_{2}+\Delta x_{d} i_{d}+\frac{P_{e}}{i_{q}}
$$

The new DFL compensated model of a power system can be generalized as in (17) where

$$
A=\left[\begin{array}{ccc}
0 & 1 & 0 \\
0 & -\frac{D}{2 H} & -\frac{\omega_{0}}{2 H} \\
0 & \frac{e_{q}^{,} V_{\infty}^{\cos \delta}}{x_{d \Sigma}^{\prime}} & 0
\end{array}\right] \quad B=\left[\begin{array}{c}
0 \\
0 \\
\frac{1}{T_{d o}^{\prime}} i_{q}
\end{array}\right]
$$

and $v_{2}$ is chosen as the new input through the excitation loop of the generator.

\subsection{Nonlinear Control Design 3}

Differentiating the active electrical power equation gives (15). Rewriting it and multiplying out the terms gives

$$
\begin{aligned}
\dot{P}_{e}= & -\frac{1}{T_{d o}^{\prime}} P_{e}+\frac{1}{T_{d o}^{\prime}} i_{q}\left(E_{f}-\Delta x_{d} i_{d}\right) \\
& +\frac{e_{q}^{\prime} V_{\infty} \cos \delta}{x_{d \Sigma}^{\prime}}\left(\omega-\omega_{0}\right) \\
= & -\frac{1}{T_{d o}^{\prime}} \Delta P_{e}+\frac{1}{T_{d o}^{\prime}} i_{q}\left(E_{f}-\Delta x_{d} i_{d}\right) \\
& +\frac{e_{q}^{\prime} V_{\infty} \cos \delta}{x_{d \Sigma}^{\prime}} \Delta \omega-\frac{1}{T_{d o}^{\prime}} P_{m}
\end{aligned}
$$

where $\Delta P_{e}=P_{e}-P_{m} ; \Delta \omega=\omega-\omega_{0}$.
If we let

we have

$$
v_{3}=i_{q}\left(E_{f}-\Delta x_{d} i_{d}\right)-P_{m}
$$

$$
\dot{P}_{e}=-\frac{1}{T_{d o}^{\prime}} \Delta P_{e}+\frac{e_{q}^{,} V_{\infty} \cos \delta}{x_{d \Sigma}^{\prime}} \Delta \omega+\frac{1}{T_{d o}^{\prime}} v_{3}
$$

Then we can get the DFL compensating law from (27) as

$$
E_{f}=\frac{1}{i_{q}}\left(v_{3}+P_{m}\right)+\Delta x_{d} i_{d}
$$

and the new DFL compensated model of a power system can be generalized as in (17) where

$$
A=\left[\begin{array}{ccc}
0 & 1 & 0 \\
0 & -\frac{D}{2 H} & -\frac{\omega_{0}}{2 H} \\
0 & \frac{e_{q}^{\prime} V_{\infty}^{\cos \delta}}{x_{d \Sigma}^{\prime}} & -\frac{1}{T_{d o}^{\prime}}
\end{array}\right] \quad B=\left[\begin{array}{c}
0 \\
0 \\
\frac{1}{T_{d o}^{\prime}}
\end{array}\right]
$$

and $v_{3}$ is chosen as the new input through the excitation loop of the generator.

\subsection{Nonlinear Control Design 4}

For the purpose of illustration and comparison, we consider the nonlinear transient stability controller proposed in Zhu et al. (1997); Guo et al. (2001). We can rewrite (26) as

$$
\dot{P}_{e}=-\frac{1}{T_{d o}^{,}} \Delta P_{e}+\frac{1}{T_{d o}^{\prime}} v_{4}
$$

where the new nonlinear input is

$$
v_{4}=i_{q}\left(E_{f}-\Delta x_{d} i_{d}\right)-P_{m}+T_{d o} P_{e} \cot \delta \Delta \omega
$$

and the DFL compensated system can be placed into state space form (17) where

$$
A=\left[\begin{array}{ccc}
0 & 1 & 0 \\
0 & -\frac{D}{2 H} & -\frac{\omega_{0}}{2 H} \\
0 & 0 & -\frac{1}{T_{d o}^{\prime}}
\end{array}\right] \quad B=\left[\begin{array}{c}
0 \\
0 \\
\frac{1}{T_{d o}^{\prime}}
\end{array}\right]
$$

and $v_{4}$ is chosen as the new input through the excitation loop of the generator. From (32) we get the DFL compensating law

$$
E_{f}=\frac{1}{i_{q}}\left(v_{4}+P_{m}-T_{d o}^{,} P_{e} \cot \delta \Delta \omega\right)+\Delta x_{d} i_{d}
$$

\subsection{Realization of Nonlinear Controllers}

We have designed different control strategies to bring out the effectiveness in maintaining the transient stability of a power system. Four new nonlinear controllers are proposed, they are

$$
\begin{aligned}
& v_{1}=i_{q} \frac{1}{T_{d o}^{\prime}}\left(E_{f}-\Delta x_{d} i_{d}-\frac{P_{e}}{i_{q}}\right)+P_{e} \cot \delta \Delta \omega \\
& v_{2}=\left(E_{f}-\Delta x_{d} i_{d}-\frac{P_{e}}{i_{q}}\right) \\
& v_{3}=i_{q}\left(E_{f}-\Delta x_{d} i_{d}\right)-P_{m} \\
& v_{4}=i_{q}\left(E_{f}-\Delta x_{d} i_{d}\right)-P_{m}+T_{d o} P_{e} \cot \delta \Delta \omega
\end{aligned}
$$




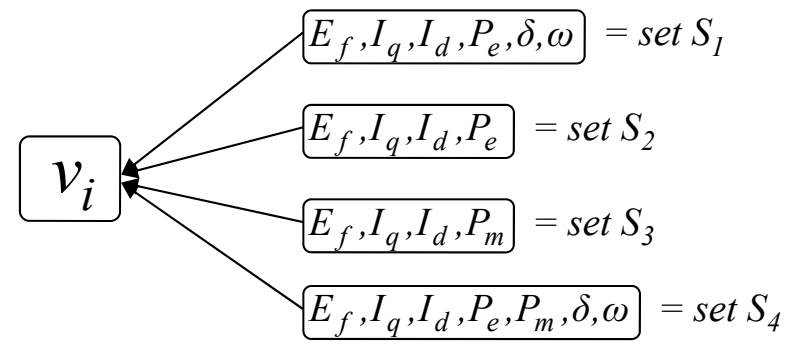

Fig. 1. Signal realization for nonlinear controllers

Fig.1 shows signal sets needed to realize each of the nonlinear control inputs given by (35)-(38) and represented by $v_{i}$ in Fig.1. We can see that signal sets $S_{1}$ and $S_{4}$ require more information to realize the nonlinear control input for controllers (35) and (38) respectively, whereas signal sets $S_{2}$ and $S_{3}$ need only few variables to realize control inputs given by (36) and (37). The proposed method enables control engineer to choose from any of these controllers based on required simplicity or availability of measurements.

\section{ROBUST CONTROL METHOD}

In this section we extend the general robust control method presented by Petersen and Hollot (1986) to consider uncertainty in both $A$ and $B$ connection matrices in designing robust excitation controller. The state space model of the DFL linearized system containing uncertainties can be described by the following state equation

$$
\dot{x}=\left(A+\sum_{i=1}^{k} A_{i} r_{i}(t)\right) x+\left(B+\sum_{i=1}^{l} B_{i} s_{i}(t)\right) u
$$

where $x(t) \in \Re^{n}$ is the state, $u(t) \in \Re^{m}$ is the control and $r(t) \in \Re^{k}, s(t) \in \Re^{l}$ represent uncertainty inputs. It has been assumed that each uncertainty parameter satisfies the same bound in the known compact sets $\mathcal{R}$ and $\mathcal{S}$ such that

$$
\begin{aligned}
& \mathcal{R}=\left\{r:\left|r_{i}\right| \leq \bar{r}, i=1,2, \ldots, k\right\} \\
& \mathcal{S}=\left\{s:\left|s_{i}\right| \leq \bar{s}, i=1,2, \ldots, l\right\}
\end{aligned}
$$

The matrices $A_{i}$ and $B_{i}$ are assumed to be rank one decompositions of the form

$$
A_{i}=d_{i} e_{i}^{T} \quad B_{i}=f_{i} g_{i}^{T}
$$

We choose the required stabilizing control law as:

$$
u(t)=-\frac{1}{\epsilon} R^{-1} B^{T} \operatorname{Px}(t)
$$

and construct a closed loop system such that

$$
\begin{aligned}
& A^{T} P+P A+\bar{r} \sum_{i=1}^{k} A_{i}^{T} P+\bar{r} P \sum_{i=1}^{k} A_{i} \\
& -\frac{2}{\epsilon} P B R^{-1} B^{T} P-\frac{1}{\epsilon} \bar{r} P B R^{-1} \sum_{i=1}^{l} B_{i}^{T} P \\
& -\frac{1}{\epsilon} \bar{r} P \sum_{i=1}^{l} B_{i} R^{-1} B^{T} P=-\epsilon Q
\end{aligned}
$$

where $\epsilon>0, Q$ and $R$ are positive definite matrices chosen by the user. Consider the fact that

$$
2 x^{T} P \Omega x=x^{T}\left(P \Omega+\Omega^{T} P\right) x
$$

and for any pair of real numbers $a$ and $b$, the following inequality holds

$$
2|a b| \leq a^{2}+b^{2}
$$

Then, using the property of (42), (45) and (46), terms on the left hand side of (44) can be rewritten

$$
\begin{aligned}
2 \bar{r} \sum_{i=1}^{l} x^{T} P d_{i} e^{T} x & \leq \bar{r} \sum_{i=1}^{k}\left(x^{T} P d_{i}\right)^{2}+\bar{r} \sum_{i=1}^{k}\left(e_{i}^{T} x\right)^{2} \\
& =\bar{r} x^{T} P \mathscr{D} P x+\bar{r} x^{T} \mathscr{E} x
\end{aligned}
$$

and also

$$
\begin{aligned}
& \frac{2}{\epsilon} \bar{s} \sum_{i=1}^{l}\left|x^{T} P f_{i} g_{i}^{T} R^{-1} B^{T} P x\right| \\
& \leq \frac{1}{\epsilon} \bar{s} \sum_{i=1}^{l}\left(x^{T} P f_{i}\right)^{2}+\frac{1}{\epsilon} \bar{s} \sum_{i=1}^{l}\left(g_{i}^{T} R^{-1} B^{T} P x\right)^{2} \\
& =\frac{\bar{s}}{\epsilon} x^{T} P \mathscr{F} P x+\frac{\bar{s}}{\epsilon} x^{T} P B R^{-1} \mathscr{G} R^{-1} B^{T} P x
\end{aligned}
$$

where

$$
\begin{gathered}
\mathscr{D}=\sum_{i=1}^{k} d_{i} d_{i}^{T} \\
\mathscr{E}=\sum_{i=1}^{k} e_{i} e_{i}^{T} \\
\mathscr{F}=\sum_{i=1}^{l} f_{i} f_{i}^{T} \\
\mathscr{G}=\sum_{i=1}^{l} g_{i} g_{i}^{T}
\end{gathered}
$$

Using (44), (47), (48), and choosing positive definite matrix $Q$ and $\epsilon>0$, then there exists positive definite solution $P$ which satisfies the following Riccati equation

$$
\begin{aligned}
& A^{T} P+P A \\
& -P\left[\frac{1}{\epsilon}\left(2 B R^{-1} B^{T}-\bar{s} B R^{-1} \mathscr{G} R^{-1} B^{T}-\bar{s} \mathscr{F}\right)-\bar{r} \mathscr{D}\right] P \\
& +\bar{r} \mathscr{E}+\epsilon Q=0
\end{aligned}
$$

\subsection{Stability Control 1}

In order to illustrate the problem of the proposed DFL controller in Section 3.1, we select the penalty matrices $R=1, Q=\operatorname{diag}[490,100,5800]$ and solve the standard LQR control problem with the optimal feedback gain

$$
K=R^{-1} B^{T} P
$$

such that

$$
v_{1}=-K x
$$

where 


$$
A=\left[\begin{array}{ccc}
0 & 1 & 0 \\
0 & -0.625 & -39.26 \\
0 & 0 & 0
\end{array}\right] \quad B=\left[\begin{array}{l}
0 \\
0 \\
1
\end{array}\right]
$$

to obtain the following DFL controller

$$
v_{1}=22.13 \Delta \delta+12.65 \Delta \omega-82.42 \Delta P_{e}
$$

\subsection{Robust Stability Control 2}

Since the uncertain functions are involved in (24), robust control design is applied to find the feedback control gain of $v_{2}$ in (21). Considering the uncertainty in the matrix structure (24), we choose the following rank one decompositions (42) as follows

$$
\begin{array}{r}
d_{1}=\left[\begin{array}{lll}
0 & 0 & 1
\end{array}\right]^{T}, e_{1}=\left[\begin{array}{lll}
0 & 1 & 0
\end{array}\right]^{T} \\
f_{1}=1, g_{1}=\left[\begin{array}{lll}
0 & 0 & 1
\end{array}\right]
\end{array}
$$

and select $R=1.1, Q=\operatorname{diag}[195,1.5,220], \epsilon=0.01$, $|\bar{r}| \leq 0.4$ and $|\bar{s}| \leq 0.01$ where

$$
A=\left[\begin{array}{ccc}
0 & 1 & 0 \\
0 & -0.625 & -39.26 \\
0 & 0.906 & 0
\end{array}\right] \quad B=\left[\begin{array}{c}
0 \\
0 \\
0.094
\end{array}\right]
$$

to solve the Riccati equation (53) and obtain the positive definite solution $P$ to form the nonlinear robust excitation control law

$$
v_{2}=22.59 \Delta \delta+7.97 \Delta \omega-82.98 \Delta P_{e}
$$

\subsection{Robust Stability Control 3}

Consider the DFL compensated power plant model in Section 3.3 where

$$
A=\left[\begin{array}{ccc}
0 & 1 & 0 \\
0 & -0.625 & -39.26 \\
0 & 0.906 & -0.144
\end{array}\right] \quad B=\left[\begin{array}{c}
0 \\
0 \\
0.144
\end{array}\right]
$$

We choose the penalty matrices $R=0.15, Q=$ $\operatorname{diag}[125,50,700]$, other relevant parameters $\epsilon=0.1,|\bar{r}| \leq$ 0.4 and $|\bar{s}|=0$ and define uncertainty decompositions as

$$
d_{1}=\left[\begin{array}{lll}
0 & 0 & 1
\end{array}\right]^{T}, e_{1}=\left[\begin{array}{lll}
0 & 1 & 0
\end{array}\right]^{T}
$$

Solving the algebraic Riccati equation (53) we obtain the robust excitation control law

$$
v_{3}=22.07 \Delta \delta+12.91 \Delta \omega-82.14 \Delta P_{e}
$$

\subsection{Robust Stability Control 4}

The DFL compensating plant model in Section 3.4 has been proposed in Zhu et al. (1997) and the transient stability controller (32) is used here for comparative purposes. The robust excitation controller is given as

$$
v_{4}=22.36 \Delta \delta+12.81 \Delta \omega-82.45 \Delta P_{e}
$$

\section{SIMULATIONS}

Following the design procedure of Section 3 and Section 4 we obtain complete nonlinear controllers:

Control 1

$$
\begin{aligned}
E_{f} & =\frac{T_{d o}^{\prime}}{i_{q}}\left(v_{1}-P_{e} \cot \delta \Delta \omega\right)+\Delta x_{d} i_{d}+\frac{P_{e}}{i_{q}} \\
v_{1} & =22.13 \Delta \delta+12.65 \Delta \omega-82.42 \Delta P_{e}
\end{aligned}
$$

Control 2

$$
\begin{aligned}
E_{f} & =v_{2}+\Delta x_{d} i_{d}+\frac{P_{e}}{i_{q}} \\
v_{2} & =22.59 \Delta \delta+7.97 \Delta \omega-82.98 \Delta P_{e}
\end{aligned}
$$

Control 3

$$
\begin{aligned}
E_{f} & =\frac{1}{i_{q}}\left(v_{3}+P_{m}\right)+\Delta x_{d} i_{d} \\
v_{3} & =22.07 \Delta \delta+12.91 \Delta \omega-82.14 \Delta P_{e}
\end{aligned}
$$

Control 4

$$
\begin{gathered}
E_{f}=\frac{1}{i_{q}}\left(v_{4}+P_{m}-T_{d o}, P_{e} \cot \delta \Delta \omega\right)+\Delta x_{d} i_{d} \\
v_{4} \quad=22.36 \Delta \delta+12.81 \Delta \omega-82.45 \Delta P_{e}
\end{gathered}
$$

Transient stability study is carried out on a simple SMIB power system model described in Section 2. The power system responses with different controllers subjected to different disturbances are given in Fig.2 and Fig.3.

Simulation results show that the proposed nonlinear controllers can effectively enhance the transient stability of a power system, even in the presence of large operating point change due to a severe short circuit fault near the generator terminals.

\section{CONCLUSIONS}

In this paper we have presented the flexibility of DFL as an appealing and simple nonlinear control tool. We have proposed and compared four different nonlinear feedback designs used to cancel the inherent power system nonlinearities and enhance the dynamic performance of the nonlinear power system. The DFL technique can be used to linearize the system over a very wide range. In the case of imperfections such as the presence of nonlinearities or uncertainties in the DFL compensated plant model, robust control theory can be used to design the robust feedback controller. We have shown that the control implementation can vary from a simple to a more complex control loop. The DFL technique presented offers a choice of different nonlinear controllers, considering the issues such as compensator complexity and availability of measurements to achieve performance enhancing stability control.

The procedure presented can be applied to other dynamical networks where the flexibility in designing the complexity of the nonlinear compensating loop is critical. Some applications for the future may include but not necessarily limited to multimachine power systems, automotive industry, robot control, biomedical applications, control of process systems. 

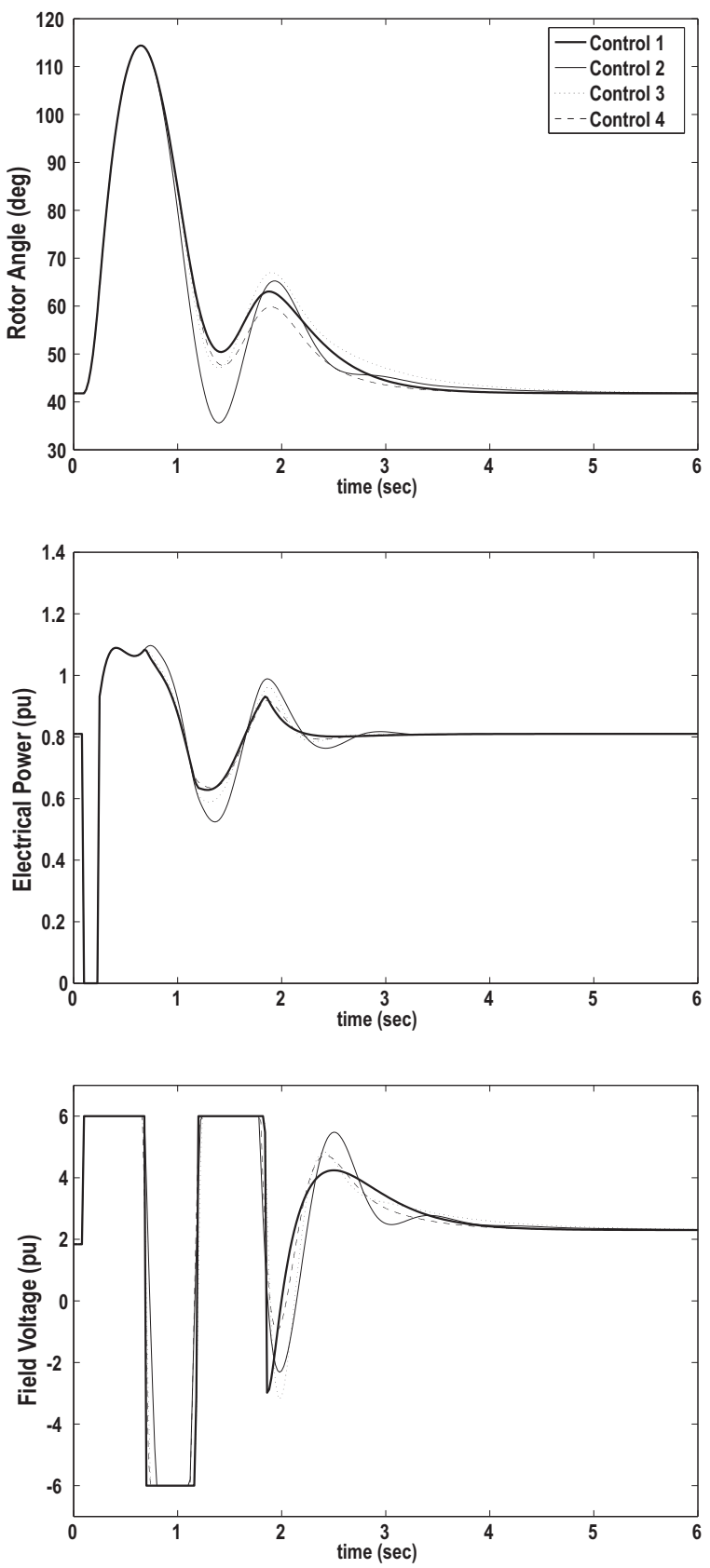

Fig. 2. Power System Responses, Disturbance 1

\section{REFERENCES}

J.W. Chapman, M.D. Ilic, C.A. King, L. Eng, and H. Kaufman. Stabilizing a multimachine power system via decentralized feedback linearizing excitation control. IEEE Trans. on Power Systems, 8(3), 1993.

L. Gao, L. Chen, Y. Fan, and H. Ma. A nonlinear control design for power systems. Automatica, 28:975-979, 1992.

M. Gordon and D.J. Hill. On structure preserving control of power systems. IEEE Conf. on Control Applications, Munich, Germany, 2006.

Y. Guo, D.J. Hill, and Y. Wang. Global transient stability and voltage regulation for power systems. IEEE Trans. on Power Systems, 16(4), 2001.

A. Isidori. Nonlinear Control Systems. Communications and Control Engineering Series, Springer-Verlag, 3rd
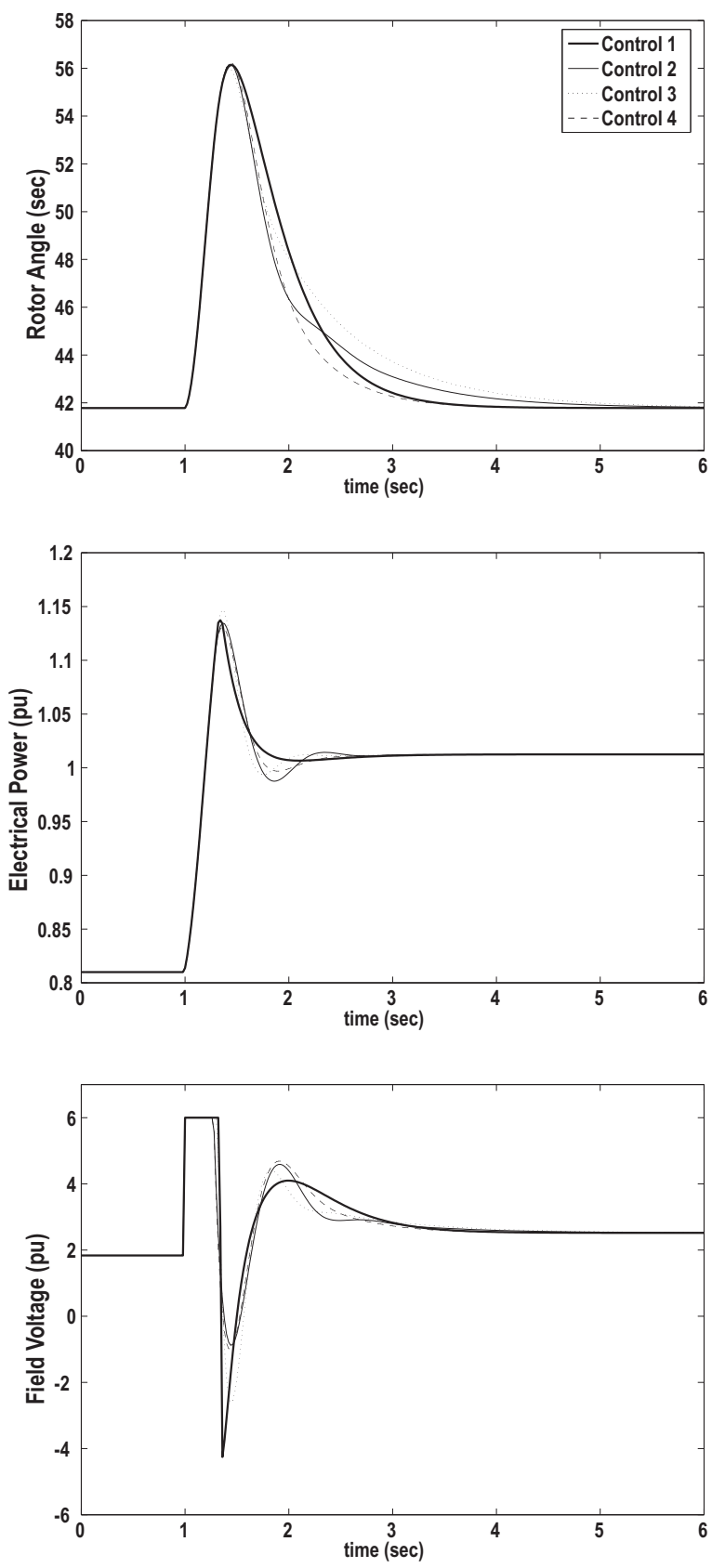

Fig. 3. Power System Responses, Disturbance 2

ed., 1995.

Q. Lu and Y.Z. Sun. Nonlinear stabilizing control of multimachine systems. IEEE Transactions on Power Systems, 4(4), 1989.

R. Marino. An example of a nonlinear regulator. IEEE Transactions on Automatic Control, 29(3):276279, 1984.

I.R. Petersen and C.V. Hollot. A Riccati equation approach to the stabilization of uncertain linear systems. Automatica, 22(4):397-411, 1986.

Y. Wang, D.J. Hill, R.H. Middleton, and L. Gao. Transient stability enhancement and voltage regulation of power systems. IEEE Trans. on Power Systems, 8(2), 1993.

C. Zhu, R. Zhou, and Y. Wang. A new nonlinear voltage controller for power systems. Electrical Power and Energy Systems, 19(1):19-27, 1997. 